\title{
A Note on the Divergence-Free Jacobian Conjecture in $\mathbb{R}^{2}$
}

\author{
by \\ M. SABATINI \\ Presented by Czesław OLECH
}

Summary. We give a shorter proof to a recent result by Neuberger [Rocky Mountain J. Math. 36 (2006)], in the real case. Our result is essentially an application of the global asymptotic stability Jacobian Conjecture. We also extend some of the results of Neuberger's paper.

1. Introduction. The classical Jacobian Conjecture was formulated in [8] as a problem about the global invertibility of polynomial maps $\Phi: \mathbb{C}^{n}$ $\rightarrow \mathbb{C}^{n}$. Keller asked whether a polynomial map with constant non-zero Jacobian determinant is globally invertible, and whether its inverse is also a polynomial map. The problem was widely studied in subsequent decades, producing several partial results and even some faulty proofs. In [1] one finds a historical overview of research about the Jacobian Conjecture and a rich survey of results published up to 1982. The paper [2] contains a more recent list of results and some equivalent formulations of the problem in any dimension. Among general results concerning that problem, it is known that it is equivalent to prove or disprove the statement in any field of zero characteristic, that it is sufficient to prove $\Phi$ 's injectivity in order to get its surjectivity [1], and that $\Phi$ 's global invertibility implies that $\Phi^{-1}$ is a polynomial map. The most studied special case is the bidimensional one, $\Phi(x, y)=(P(x, y), Q(x, y))$, where the statement was proved under the hypothesis that either $P$ 's or $Q$ 's degree is 4 , or prime, or both degrees are $\leq$ 100 (see [1] for a more comprehensive list of results). A recent result, which is the object of this paper, proves the global invertibility of jacobian maps of the form $\Phi(x, y)=(x+p(x, y), y+q(x, y))$ under the additional assumptions 
that

$$
\frac{\partial p}{\partial x}+\frac{\partial q}{\partial y}=0 \quad \text { and } \quad \frac{\partial p}{\partial x} \frac{\partial q}{\partial y}-\frac{\partial p}{\partial y} \frac{\partial q}{\partial x}=0 .
$$

In higher dimensions, a striking result states that, in order to prove the $n$-dimensional Jacobian conjecture, it is sufficient to prove it for maps of the form $\Phi=L+C, L$ linear, $C$ cubic [1], or even for maps of the form $\Phi(X)=X+(A X)^{o 3}[4]$.

A different question, arising in differential equations from the study of a critical point's global stability, is also known as a Jacobian Conjecture. It is concerned with the global asymptotic stability (g.a.s.) of a critical point of a vector field whose jacobian eigenvalues have negative real part at every point of the space [10]. In [12] it was shown that under such hypotheses, it is equivalent to prove the global asymptotic stability of a critical point or the global injectivity of the vector field. Such a result gave a new direction to the research about the g.a.s. Jacobian Conjecture. Thanks also to such a new approach, the question was positively settled in dimension 2 in [5], [6], [7]. In higher dimensions the conjecture is known to be false [3], unless some additional hypotheses hold.

In this paper we give a shorter proof to Neuberger's result [11] in the real case, showing that it is actually a consequence of the bidimensional g.a.s. Jacobian Conjecture. Actually, we prove something more, since we do not make assumptions on the terms in $\frac{\partial p}{\partial x} \frac{\partial q}{\partial y}-\frac{\partial p}{\partial y} \frac{\partial q}{\partial x}$. For jacobian maps, it is sufficient to require that $\frac{\partial p}{\partial x}+\frac{\partial q}{\partial y} \geq 0$. Then we look for algebraic-like conditions which imply that property, involving the degree and order of the real polynomials $P$ and $Q$, or the degrees of the monomials contained in $P$ and $Q$. We extend some of the results proved in [11], and criticize one of them.

2. Results. Throughout this paper we only consider polynomials with real coefficients. Given a polynomial $P$, we write $d(P)$ for its degree and $o(P)$ for its order. We say that a polynomial is even if it is the sum of even-degree monomials, and odd if it is the sum of odd-degree monomials. Similarly, we say that a polynomial is $x$-even if it contains only terms with even powers of $x$, and $x$-odd if it contains only terms with odd powers of $x$.

We say that a non-negative integer is a $g a p$ of $P$ if it is the difference of the degrees of two distinct monomials in $P$. We denote by $G(P)$ the gap-set of $P$. As an example, the polynomial $P(x, y)=x^{3}+y^{3}+x^{2} y^{2}+y^{7}$ has gap-set $G(P)=\{0,1,3,4\}$. If $P$ has exactly one monomial, then we say that it has empty gap-set.

We say that the couple of polynomials $(P, Q)$ satisfies the gap condition if for every monomial $M$ in $P$, one has $d(M)-1 \notin G(Q)$. The gap condition 
is not symmetric, as shown by the couple $(P, Q)=\left(x+y^{2}, x^{6}+y^{2}\right)$. In that case one has $G(P)=\{1\}, G(Q)=\{4\}$, so that $(P, Q)$ satisfies the gap condition, but $(Q, P)$ does not.

We say that $(P, Q)$ satisfies the symmetric gap condition if both $(P, Q)$ and $(Q, P)$ satisfy the gap condition.

Let $\Phi: \mathbb{R}^{2} \rightarrow \mathbb{R}^{2}, \Phi(x, y) \equiv(P(x, y), Q(x, y))$, be a real polynomial map. Let $J_{\Phi}$ be its jacobian matrix. We say that $\Phi$ is a jacobian map if its jacobian determinant $\operatorname{det} J_{\Phi}$ is a non-zero constant. We first consider a straightforward consequence of the g.a.s. Jacobian Conjecture.

Lemma 1. Let $\Phi^{*}: \mathbb{R}^{2} \rightarrow \mathbb{R}^{2}, \Phi^{*}(u, v)=\left(u+p^{*}(u, v), v+q^{*}(u, v)\right)$, be $a$ jacobian polynomial map with $o\left(p^{*}\right), o\left(q^{*}\right)>1$. If

then $\Phi^{*}$ is injective.

$$
\frac{\partial p^{*}}{\partial u}+\frac{\partial q^{*}}{\partial v} \geq 0
$$

Proof. Since det $J_{\Phi^{*}}$ is constant, its value can be evaluated at the origin, hence det $J_{\Phi^{*}}=1$. Let us consider the planar differential system associated to the map $-\Phi^{*}$,

$$
\dot{u}=-u-p^{*}(u, v), \quad \dot{v}=-v-q^{*}(u, v) .
$$

Its jacobian matrix is $-J_{\Phi^{*}}$. Since $\partial p^{*} / \partial u+\partial q^{*} / \partial v \geq 0$, at every point of the plane $-J_{\Phi^{*}}$ has trace $\leq-2<0$, and determinant $\operatorname{det}\left(-J_{\Phi^{*}}\right)=\operatorname{det} J_{\Phi^{*}}=$ $1>0$, hence its eigenvalues have negative real part at every point of $\mathbb{R}^{2}$. Since the g.a.s. Jacobian Conjecture holds, $-\Phi^{*}$ is injective, hence so is $\Phi^{*}$.

Neuberger's result for real maps is contained in Lemma 1, since in [11] only maps with $\partial p^{*} / \partial u+\partial q^{*} / \partial v=0$ are considered.

In relation to the classical Jacobian Conjecture, algebraic-like hypotheses are usually considered. In fact, even if it can sometimes be checked whether $\partial p^{*} / \partial u+\partial q^{*} / \partial v \geq 0$, assumptions relating to the map's degree or order are desirable.

In [11], the injectivity of a complex jacobian map of the form $\Phi^{*}(u, v)=$ $\left(u+p^{*}(u, v), v+q^{*}(u, v)\right)$ is stated assuming the nonlinear terms in $p^{*}$ and $q^{*}$ have degrees in the interval $[n, m]$ with $(n-1)^{2}>m$. The motivation given, "there is no term in $\frac{\partial p^{*}}{\partial u} \frac{\partial q^{*}}{\partial v}-\frac{\partial p^{*}}{\partial v} \frac{\partial q^{*}}{\partial u}$ which has a degree in common with a term of $\frac{\partial p^{*}}{\partial u}+\frac{\partial q^{*}}{\partial v}$, does not hold in general. In fact, the map

$$
\Phi^{*}(u, v)=\left(u+3 u v+u^{3}, v+u^{2} / 2+v^{3}\right)
$$

has

$$
\begin{gathered}
\frac{\partial p^{*}}{\partial u}+\frac{\partial q^{*}}{\partial v}=3 v+3 u^{2}+3 v^{2}, \\
\frac{\partial p^{*}}{\partial u} \frac{\partial q^{*}}{\partial v}-\frac{\partial p^{*}}{\partial v} \frac{\partial q^{*}}{\partial u}=9 v^{3}+9 u^{2} v^{2}-3 u^{2} .
\end{gathered}
$$


The terms $3 u^{2}$ and $-3 u^{2}$ cancel with each other. This map does not have constant jacobian determinant, but in [11] it is not proved how the jacobian condition leads to the statement. Most probably the hypothesis $(n-1)^{2}>m$ should be replaced by $2(n-1)>m$, in the same spirit as in condition (i) in the next theorem.

Condition (ii) of the next theorem has been added only because we do not assume the linear part of $\Phi$ to be the identity, but the argument is the same as in [11]. The other conditions, as well as those in Theorem 2, are new.

TheOREM 1. Let $\Phi: \mathbb{R}^{2} \rightarrow \mathbb{R}^{2}$ be a jacobian map of the type $\Phi(x, y)=$ $(a x+b y+p(x, y), c x+d y+q(x, y))$ with $a, b, c, d \in \mathbb{R}$ and $o(p), o(q)>1$. If one of the following holds:

(i) $\max \{d(p), d(q)\}<o(p)+o(q)-1$,

(ii) both $p(x, y)$ and $q(x, y)$ are even polynomials,

(iii) $(p, q)$ satisfies the symmetric gap condition,

then $\Phi$ is globally invertible.

Proof. Without loss of generality, we may assume $a d-b c>0$. Let $A$ be the linear map associated to the matrix

$$
\left(\begin{array}{ll}
a & b \\
c & d
\end{array}\right) .
$$

Let $A^{-1}$ be its inverse. Set $\Phi^{*}(u, v)=\Phi\left(A^{-1}(u, v)\right)$. The linear part of $\Phi^{*}$ is just the composition of $A^{-1}$ and $A$, hence it is the identity. Thus, $\Phi^{*}(u, v)=$ $\left(u+p^{*}(u, v), v+q^{*}(u, v)\right)$ with $\operatorname{det} J_{\Phi^{*}}=$ const $>0$. A linear change of variables does not change a polynomial's order, its degree and the property of being even or odd, as above defined. Hence $o\left(p^{*}\right), o\left(q^{*}\right)>1$, and conditions (i)-(iv) hold for $p^{*}$ and $q^{*}$ as well. Moreover, $\operatorname{det} J_{\Phi^{*}}=$ const $>0$. Without loss of generality we may assume $\operatorname{det} J_{\Phi^{*}}=1$.

Computing the jacobian determinant of $\Phi^{*}$ gives

$$
\operatorname{det} J_{\Phi^{*}}=1+\left(\frac{\partial p^{*}}{\partial u}+\frac{\partial q^{*}}{\partial v}\right)+\left(\frac{\partial p^{*}}{\partial u} \frac{\partial q^{*}}{\partial v}-\frac{\partial p^{*}}{\partial v} \frac{\partial q^{*}}{\partial u}\right) .
$$

Set

$$
T^{*}=\frac{\partial p^{*}}{\partial u}+\frac{\partial q^{*}}{\partial v}, \quad D^{*}=\frac{\partial p^{*}}{\partial u} \frac{\partial q^{*}}{\partial v}-\frac{\partial p^{*}}{\partial v} \frac{\partial q^{*}}{\partial u} .
$$

Since $o\left(T^{*}+D^{*}\right)>0$, one has $T^{*}+D^{*} \equiv 0$.

Under assumption (i), observe that the highest degree monomial in $T^{*}$ has degree $\leq \max \{d(p), d(q)\}-1$, while the lowest degree monomial in $D^{*}$ has degree $\geq o(p)+o(q)-2$. If $\max \{d(p), d(q)\}<o(p)+o(q)-1$, then $T^{*}$ and $D^{*}$ have no monomials of the same degree, hence, from $T^{*}+D^{*} \equiv 0$, 
one gets both $T^{*} \equiv 0$ and $D^{*} \equiv 0$. Applying Lemma 1 one proves that $-\Phi^{*}$ is injective, hence so are $\Phi^{*}$ and $\Phi$.

Now, under assumption (ii), note that if both $p(x, y)$ and $q(x, y)$ are even polynomials, then $p^{*}(x, y)$ and $q^{*}(x, y)$ are even, $T^{*}$ is odd and $D^{*}$ is even. From $T^{*}+D^{*} \equiv 0$, one gets again $T^{*} \equiv 0$ and $D^{*} \equiv 0$, since monomials of $T^{*}$ do not cancel with monomials of $D^{*}$. Then one can proceed as in (i) to get the injectivity of $\Phi$.

Under the hypothesis of (iii), assume for contradiction that there exists a positive integer $h$ such that both $\partial q^{*} / \partial v$ and $D^{*}$ have a monomial of degree $h$. Then $q$ has a monomial $M$ such that $d(M)-1=h$. Also, there exist monomials $K$ in $p$ and $L$ in $q$ such that $d(K)+d(L)-2=h$. Hence $d(M)-d(L)=d(K)-1$, contradicting the gap condition. Similarly, one gets a contradiction assuming the existence of a positive integer $h$ such that both $\partial p^{*} / \partial u$ and $D^{*}$ have a monomial of degree $h$. This proves that $T^{*}$ and $D^{*}$ have no monomials of the same degree, so that $T^{*} \equiv 0$ and $D^{*} \equiv 0$. Then the above argument applies.

In order to show that we are considering non-empty hypotheses, we give some examples of jacobian mappings satisfying the above conditions. For condition (i) we may choose Meisters' maps,

$\Phi(x, y)=\left(a x+b y+\mu(\alpha a+\beta b)(\alpha y-\beta x)^{2}, c x+d y+\mu(\alpha c+\beta d)(\alpha y-\beta x)^{2}\right)$ with $\mu \neq 0,(\alpha, \beta) \neq(0,0), a d-b c \neq 0$. For condition (ii) we may consider

$$
\Phi(x, y)=\left(x+y+x^{4}+x^{6}, y+x^{4}+x^{6}\right) .
$$

An example of a map satisfying condition (iii) is

$$
\Phi(x, y)=\left(x+(x-y)^{3}+(x-y)^{10}, y+(x-y)^{3}+(x-y)^{10}\right) .
$$

In [1] it was proved that in order to prove the Jacobian Conjecture it is sufficient to prove it for cubic-linear jacobian maps. It may be interesting to show that a simple consequence of the above theorem is the invertibility of linear + homogeneous jacobian real maps.

Corollary 1. Let $\Phi: \mathbb{R}^{2} \rightarrow \mathbb{R}^{2}$ be a jacobian map of the type $\Phi(x, y)=$ $\left(a x+b y+p_{n}(x, y), c x+d y+q_{n}(x, y)\right), a, b, c, d \in \mathbb{R}$, with $p_{n}$ and $q_{n}$ homogeneous polynomials of the same degree $n>1$. Then $\Phi$ is globally invertible.

Proof. One has $o(p)=o(q)=d(p)=d(q)=n$, hence condition (i) is satisfied: $\max \{d(p), d(q)\}=n<2 n-1=o(p)+o(q)-1$.

An example of a planar linear + cubic jacobian map is

$$
\begin{aligned}
P(x, y) & =2 x-y+x^{3}+x^{2} y+\frac{x y^{2}}{3}+\frac{y^{3}}{27}, \\
Q & =3 x-3 y+\frac{12 x^{3}}{5}+\frac{12 x^{2} y}{5}+\frac{4 x y^{2}}{5}+\frac{4 y^{3}}{45} .
\end{aligned}
$$


A result slightly different from Theorem 1 can be proved by assuming different symmetries on $p$ and $q$. In the next statement we assume $\Phi$ to be of the form $(x+p(x, y), y+q(x, y))$, as in [11], since a linear change of variables in general does not preserve the required symmetry property.

TheOREM 2. Let $\Phi: \mathbb{R}^{2} \rightarrow \mathbb{R}^{2}$ be a jacobian map of the type $\Phi(x, y)=$ $(x+p(x, y), y+q(x, y)), o(p), o(q)>1$. If one of the following holds:

(i) $p$ is $x$-even, $q$ is $x$-odd,

(ii) $p$ is $y$-odd, $q$ is $y$-even,

then $\Phi$ is globally invertible.

Proof. Working as in Theorem 1, one has

$$
\operatorname{det} J_{\Phi}=1+\left(\frac{\partial p}{\partial x}+\frac{\partial q}{\partial y}\right)+\left(\frac{\partial p}{\partial x} \frac{\partial q}{\partial y}-\frac{\partial p}{\partial y} \frac{\partial q}{\partial x}\right) .
$$

Set

$$
T=\frac{\partial p}{\partial x}+\frac{\partial q}{\partial y}, \quad D=\frac{\partial p}{\partial x} \frac{\partial q}{\partial y}-\frac{\partial p}{\partial y} \frac{\partial q}{\partial x} .
$$

As in Theorem 1, since $o(T+D)>0$, one has $T+D \equiv 0$.

If (i) holds, then $T$ is $x$-odd and $D$ is $x$-even. Hence terms of $T$ and $D$ cannot cancel with each other, and both $T$ and $D$ vanish identically. Then one can proceed as in the proof of Theorem 1.

If (ii) holds, then $T$ is $y$-odd and $D$ is $y$-even. Then one can proceed as above.

Acknowledgements. The author would like to thank Prof. M. Sala, of the Univ. of Trento, for several conversations about the Jacobian Conjecture.

This research was partially supported by the PRIN group "Dinamica anolonoma, hamiltoniana, delle popolazioni e dei sistemi piani" and by the GNAMPA group "Analisi qualitativa e comportamento asintotico di equazioni differenziali ordinarie e di equazioni alle differenze".

\section{References}

[1] H. Bass, E. H. Connell and D. Wright, The Jacobian conjecture: reduction of degree and formal expansion of the inverse, Bull. Amer. Math. Soc. 7 (1982), 287-330.

[2] M. de Bondt and A. van den Essen, Recent progress on the Jacobian Conjecture, Bull. Amer. Math. Soc. 7 (1982), 287-330.

[3] A. Cima, A. van den Essen, A. Gasull, E. Hubbers and F. Manyosas, A polynomial counterexample to the Markus-Yamabe conjecture, Adv. Math. 131 (1997), 453-457.

[4] L. Drużkowski, An effective approach to Keller's Jacobian conjecture, Math. Ann. 264 (1983), 303-313.

[5] R. Fessler, A proof of the two-dimensional Markus-Yamabe stability conjecture and a generalization, Ann. Polon. Math. 62 (1995), 45-74. 
[6] A. A. Glutsyuk, Complete solution of the Jacobian problem for planar vector fields, Uspekhi Mat. Nauk 49 (1994), no. 3, 179-180 (in Russian); English transl.: Russian Math. Surveys 49 (1994), 185-186.

[7] C. Gutiérrez, A solution to the bidimensional global asymptotic stability conjecture, Ann. Inst. H. Poincaré Anal. Non Linéaire 12 (1995), 627-671.

[8] O. H. Keller, Ganze Cremona-Transformationen, Monatsh. Math. Phys. 47 (1939), 299-306.

[9] G. Meisters, Jacobian problems in differential equations and algebraic geometry, Rocky Mountain J. Math. 12 (1982), 679-705.

[10] L. Markus and H. Yamabe, Global stability criteria for differential systems, Osaka Math. J. 12 (1960), 305-317.

[11] J. W. Neuberger, The divergence-free Jacobian conjecture in dimension two, Rocky Mountain J. Math. 36 (2006), 265-271.

[12] C. Olech, On the global stability of an autonomous system in the plane, Contributions to Differential Equations 1 (1963), 389-400.

M. Sabatini

Departimento di Matematica

Università di Trento

I-38050 Povo (TN), Italy

E-mail: marco.sabatini@unitn.it

Received March 30, 2009;

received in final form July 22, 2009 